Contemporary Engineering Sciences, Vol. 9, 2016, no. 4, 175 - 185

HIKARI Ltd, www.m-hikari.com

http://dx.doi.org/10.12988/ces.2016.512319

\title{
Parameters Estimation in Mechanism Design
}

\author{
Yury Pichugin \\ Department of Mathematics and Mechanics \\ State University of Aerospace Instrumentation \\ Saint-Petersburg, Russia, 19000 \\ Gennadiy Alferov \\ Applied Mathematics and Control Processes \\ Saint-Petersburg State University \\ Saint-Petersburg, Russia, 198504 \\ Oleg Malafeyev \\ Applied Mathematics and Control Processes \\ Saint-Petersburg State University \\ Saint-Petersburg, Russia, 198504
}

Copyright (c) 2015 Yury Pichugin, Gennadiy Alferov and Oleg Malafeyev. This article is distributed under the Creative Commons Attribution License, which permits unrestricted use, distribution, and reproduction in any medium, provided the original work is properly cited.

\begin{abstract}
The paper proposes a procedure to estimate the parameters of experimental manipulator robot samples in a series of tests that allows discarding robot samples with parameters being unsuitable for the constructed mechanism. Features of this approach, providing numerical estimates of the parameters are particularly useful in solving the problems of designing space manipulators that require a higher degree of reliability performance. This procedure can also be used in other practice areas, such as analysis of indirect signs of corruption in the socioeconomic, financial, military, geopolitical and other structures.
\end{abstract}

Keywords: mechanism, manipulation robot, stability, statistics 


\section{Introduction}

An important stage in the process of machinery design and construction is one of selecting their parameters by ad- equate performance characteristics, as well as manufacturing and testing an experimental sample of its accordance with the required characteristics. It means the parameters of the designed mechanism should meet a set of requirements, consistent with the problems which are supposed to be solved by the constructed mechanism $[3,8,14,15$, 16]. This paper proposes a procedure for estimating the parameters of the experimental sample in a series of tests to reject samples with unsuitable for the constructed mechanism parameters. The prerequisites for the use of this procedure is the assumption about multivariate normal distribution of test results, as well as the assumption about opportunities for analytical description of the domain boundaries of admissible values from the estimated parameters vector in accordance with the technical requirements. The procedure is based on the geometric properties of the normal distribution. Indeed, suppose it is required to test a complex hypothesis that the parameter vector is in a given domain. It follows from the geometric properties of the normal distribution that a simple hypothesis is enough to be tested. A simple hypothesis assumes that the parameter vector is a boundary point of a given domain, which is maximum value of likelihood. In this case, likelihood is calculated according to the probability distribution obtained from testing a statistical evaluation of the parameters. This procedure is multiple- purpose and can be also used in the other practice areas, such as analysis of indirect signs of corruption in the socio - economic, financial, military, geopolitical and other structures $[1,2,7,10]$.

\section{Manipulation Robots}

Manipulation robots are of important concern in everyday life and production. They are used in traditionally high- technology areas such as space robotics, medicine, military (reconnaissance aircraft, artillery, mine clearance) as well as in industry. The range of tasks performed by robots in the industry is very broad: vehicle assembly, conveyor assembly devices, welding, painting, goods transporting. Due to the market conditions it is necessary to increase the speedwork and accuracy of robot manipulators. However, increasing the speedwork of manipulation robots leads to a risk of losing their accuracy.

Various motions of manipulators are provided mainly due to the two types of kinematic mechanisms - rotational and translational motions. Length of manipulators units are selected from the condition that a characteristic point comes up to the executive body on a set position for a particular manipulation complex. 
The general problem of modeling a mechanical system is the choice of a sufficient degree of similarity between the model and the real modeled object. In our case, the object of simulation is a mechanical unit in the form of a system providing the necessary mechanical motions to perform the specified manipulation functions $[5,9]$.

When designing assemblies of manipulator robots based on mathematical calculations, the selection of optimal construc- tions should be made. According to the results of tests, the model that best meets the specified requirements is chosen. Thus, the use of mathematical modeling in the development of manipulator robots eliminates producing a large number of experimental samples.

\section{Estimation Of Manipulation Robots And Mech- anisms Parameters}

We assume that given parameters form an $m$-dimensional vector $\boldsymbol{\Theta}=\left(\theta_{\mathbf{1}}, \theta_{\mathbf{2}}, \ldots, \theta_{\mathbf{m}}\right)^{\mathrm{T}}$ ( $\mathrm{T}$ is a transposition operator). A series of tests of the experimental sample gives a set of measured values of the parameter $\boldsymbol{\Theta}_{\mathbf{j}}=\left(\theta_{\mathbf{1} \mathbf{j}}, \theta_{\mathbf{2} \mathbf{j}}, \ldots, \theta_{\mathbf{m} \mathbf{j}}\right)^{\mathrm{T}}, j=1,2, \ldots, n$, where $j$ is a serial number of the test. We also assume that the errors of parameters measurements are subject to a multivariate normal distribution. Then the estimation of the testing sample parameters $\Theta_{\mathbf{e}}$ in implemented series of tests, as well as estimation of the variances matrix and mutual covariances of components of this vector are, respectively,

$$
\Theta_{\mathrm{e}}=\mathrm{n}^{-1} \sum_{\mathbf{j}=1, \ldots, \mathrm{n}} \Theta_{\mathbf{j}}, \mathrm{V}_{\Theta}=\mathrm{n}^{-1} \mathrm{~V}
$$

where $\mathbf{V}=(\mathbf{n}-\mathbf{1})^{-\mathbf{1}} \sum_{\mathbf{j}=\mathbf{1}, \ldots, \mathbf{n}}\left(\boldsymbol{\Theta}_{\mathbf{j}}-\boldsymbol{\Theta}_{\mathbf{e}}\right)\left(\boldsymbol{\Theta}_{\mathbf{j}}-\boldsymbol{\Theta}_{\mathbf{e}}\right)^{\mathrm{T}}$

and $\sum_{j=1, \ldots, n}$ is a summation operator.

The decision on the accordance of experimental sample with the specified requirements in terms of mathematical statistics can be formulated as a hypothesis

$$
H_{0}: E \Theta_{\mathbf{e}}=\Theta_{\mathbf{0}},
$$

where $E$ is an expectation operator.

To test the hypothesis $H_{0}$ the following statistics may be used. In the stated assumptions (if the hypothesis $H_{0}$ is true) the value

$$
\gamma=\left(\boldsymbol{\Theta}_{\mathbf{e}}-\boldsymbol{\Theta}_{\mathbf{0}}\right)^{\mathrm{T}} \mathbf{V}_{\boldsymbol{\Theta}}^{-1}\left(\boldsymbol{\Theta}_{\mathbf{e}}-\boldsymbol{\Theta}_{\mathbf{0}}\right)
$$

has the distribution $\chi^{2}$ in some approximation with $m$ degrees of freedom (the larger the sample volume $n$, the closer it is). In addition, we can construct $\mathrm{m}$ 
Student statistics. Let the orthogonal matrix $\mathbf{Q}$ reduce matrix $\mathbf{V}_{\Theta}$ to diagonal form

$$
\mathbf{Q}^{\mathrm{T}} \mathbf{V}_{\Theta} \mathbf{Q}=\boldsymbol{\Lambda}=\operatorname{diag}\left(\lambda_{\mathbf{1}}, \lambda_{\mathbf{2}}, \ldots, \lambda_{\mathbf{m}}\right)
$$

then the components $s_{i}(i=1,2, \ldots, m)$ of the vector

$$
\mathrm{S}=\Lambda^{-1 / 2} \mathrm{Q}^{\mathrm{T}}\left(\Theta_{\mathrm{e}}-\Theta_{0}\right)
$$

have exactly Student distribution with degree of freedom $n-1$ under the same assumptions. Assume the value significance point $\alpha$ is set $(\alpha=0,05$ or $\alpha=$ $0,01)$. Then we can reject a testing sample if at least one of the following inequalities rejecting a hypothesis $H_{0}$ is satisfied

$$
\gamma>\chi_{m}^{2}(\alpha)
$$

or

$$
\left|s_{i}\right|>t_{n-1}^{\alpha / 2},(i=1,2, \ldots, m)
$$

where the quantities $\chi_{m}^{2}(\alpha)$ and $t_{n-1}^{\alpha / 2}$ define appropriate boundaries for rejection zone. With such a "point" formulation of requirements to the parameters we come up against a situation when we are interested to accept testable hypothesis, rather than reject. This introduces certain difficulties for proving the hypothesis, which leads to the need for artificial formulation of alternative hypotheses in some cases.

However, the condition for the acceptance of the product can be formulated in the form of requirement for a parameter vector to belong to some region $\omega$ in $m$-dimensional real space $\left(\omega \subset R^{m}\right)$. In this case we assume that the boundary $\partial \omega$ of the region is defined by system of equations $\varphi_{k}(\boldsymbol{\Theta})=\mathbf{0}, \mathbf{k}=\mathbf{1}, \mathbf{2}, \ldots, \mathbf{k}_{\omega}$, where each function $\varphi_{k}$ is differentiable in all arguments. Assume parameter estimator $\Theta_{\mathbf{e}}$ is in the region $\omega$. In this situation, to make sure an experimental sample is qualitative it is necessary to check the hypothesis $H_{c \omega}: E \boldsymbol{\Theta}_{\mathbf{e}} \in \mathbf{c} \omega$, which is equivalent to $E \boldsymbol{\Theta}_{\mathbf{e}} \notin \omega$ (where $c \omega$ is an addition to $\omega$ in $R^{m}$ ). Thus, in this situation we have composite multivariate hypothesis. To check $H_{c \omega}$ we need to find a boundary point $\Theta_{*}$ of the region $\omega$, which is the solution of Lagrange problems for Lagrangian functions defined as following:

$$
\begin{array}{r}
L_{k}(\boldsymbol{\Theta}, \lambda)=\ln \left(\mathbf{f}\left(\boldsymbol{\Theta}, \boldsymbol{\Theta}_{\mathbf{e}}, \mathbf{V}_{\boldsymbol{\Theta}}\right)\right)-\lambda \varphi_{\mathbf{k}}(\boldsymbol{\Theta}), \\
\left(k=1,2, \ldots, k_{\omega}\right)
\end{array}
$$

Here $f\left(\boldsymbol{\Theta}, \boldsymbol{\Theta}_{\mathbf{e}}, \mathbf{V}_{\boldsymbol{\Theta}}\right)$ is a density function of the multivariate normal distribution $N\left(\boldsymbol{\Theta}_{\mathbf{e}}, \mathbf{V}_{\boldsymbol{\Theta}}\right)$ of parameter vector evaluation $\boldsymbol{\Theta}_{\mathbf{e}}$. The value giving the maximum likelihood of $f\left(\boldsymbol{\Theta}_{*}, \boldsymbol{\Theta}_{\mathbf{e}}, \mathbf{V}_{\boldsymbol{\Theta}}\right)$ should be chosen as the final solution of the system 
(5) $\boldsymbol{\Theta}_{*}$ from $k_{\omega}$ options. Thus, $\boldsymbol{\Theta}_{*}$ is a boundary point of maximum likelihood for the distribution $N\left(\boldsymbol{\Theta}_{\mathbf{e}}, \mathbf{V}_{\boldsymbol{\Theta}}\right)$.

According to our assumption $\left(\boldsymbol{\Theta}_{\mathbf{e}} \in \omega\right)$, denote that a probability measure of ellipsoid insidedness

$$
W_{*}=\left\{\boldsymbol{\Theta}: \mathbf{f}\left(\boldsymbol{\Theta}, \boldsymbol{\Theta}_{\mathbf{e}}, \mathbf{V}_{\boldsymbol{\Theta}}\right)=\mathbf{f}\left(\boldsymbol{\Theta}_{*}, \boldsymbol{\Theta}_{\mathbf{e}}, \mathbf{V}_{\boldsymbol{\Theta}}\right)\right\}
$$

with distribution $N\left(\boldsymbol{\Theta}_{\mathbf{e}}, \mathbf{V}_{\boldsymbol{\Theta}}\right)$ can be considered as a probability measure of experimental sample reliability $[4,11,12,13]$. In this context the ellipsoid $W_{*}$ should be denominated as a reliability ellipsoid.

To test the composite hypothesis $H_{c \omega}$ we need to check a simple one $H_{*}$ : $\Theta_{\mathbf{e}}=\Theta_{*}$. For this we use statistics

$$
\gamma_{*}=\left(\boldsymbol{\Theta}_{\mathbf{e}}-\Theta_{*}\right)^{\mathrm{T}} \mathbf{V}_{\boldsymbol{\Theta}}{ }^{-\mathbf{1}}\left(\boldsymbol{\Theta}_{\mathbf{e}}-\boldsymbol{\Theta}_{*}\right) \text { and } \mathbf{S}_{*}=\boldsymbol{\Lambda}^{-\mathbf{1} / \mathbf{2}} \mathbf{Q}^{\mathrm{T}}\left(\boldsymbol{\Theta}_{\mathbf{e}}-\boldsymbol{\Theta}_{*}\right)
$$

This statistics can be found from statistics 1 and 2 by substituting the value $\boldsymbol{\Theta}_{*}$ instead of $\boldsymbol{\Theta}_{\mathbf{0}}$. If at least one of the inequalities $\gamma_{*}>\chi_{m}^{2}(\alpha)$ or $\left|s_{* i}\right|>$ $t_{n-1}^{\alpha / 2}, \quad(i=1,2, \ldots, m)$ is satisfied, then for some selected value $\alpha$ we reject the hypothesis $H_{*}$.

Now we show that we actually reject the hypothesis $H_{c \omega}$ by rejecting the hypothesis $H_{*}$. In other words we make sure in complete and statistically reliable accordance of experimental sample with specified requirements.

Indeed, a composite hypothesis $H_{c \omega}$ can be considered as a set of simple hypotheses $H_{\tau}: \quad E \boldsymbol{\Theta}_{\mathbf{e}}=\boldsymbol{\Theta}_{\tau}, \quad\left(\boldsymbol{\Theta}_{\tau} \in \mathbf{c} \omega\right)$. Therefore, it is enough to make sure that if the hypothesis $H_{*}$ for any value $\boldsymbol{\Theta}_{\tau}$ from region $c \omega$ is rejected, then hypothesis $H_{\tau}$ is also rejected [11]. With this end in view, we introduce a generalized Euclidean metric in space $R^{m}$ generated by the distribution $N\left(\boldsymbol{\Theta}_{\mathbf{e}}, \mathbf{V}_{\boldsymbol{\Theta}}\right)$ :

$$
d_{2}\left(\boldsymbol{\Theta}_{1}, \Theta_{2}\right)=\left[\left(\Theta_{1}-\Theta_{2}\right)^{\mathrm{T}} \mathbf{V}_{\boldsymbol{\Theta}^{-1}}\left(\boldsymbol{\Theta}_{1}-\boldsymbol{\Theta}_{\mathbf{2}}\right)\right]^{\mathbf{1} / \mathbf{2}} .
$$

Obviously in this metric the point $\boldsymbol{\Theta}_{*}$ is closer to the point $\boldsymbol{\Theta}_{\mathbf{e}}$ than any point $\boldsymbol{\Theta}_{\tau}: \boldsymbol{\Theta}_{\tau} \in \mathbf{c} \omega$, which is provided by the maximum value of the likelihood at the boundary point $\boldsymbol{\Theta}_{*}$ (see above) and a monotonic increase of the square root. It follows from the above that if the inequality (3) for statistics calculated in the point $\boldsymbol{\Theta}_{*}$ is satisfied, then this inequality is satisfied in any point $\boldsymbol{\Theta}_{\tau}$, and therefore, the hypothesis $H_{\tau}$ is rejected. For Student statistics of the form (2) and the inequalities (4) we have a similar situation, as the use of these statistics implies the transition to the equivalent metric under the metric $d_{\infty}$ (maximum of component modulus) in the space under the linear transformation with matrix operator $\boldsymbol{\Lambda}^{-\mathbf{1} / \mathbf{2}} \mathrm{Q}^{\mathrm{T}}$ (see remarks 1 and 2 ).

Remark 1. The concept of a generalized Euclidean metric is closely related with the notion of the affine space transformation, which keeps all the metric 
ratios (like the inverse transformation). Here, the linear transformation with the matrix operator $\boldsymbol{\Lambda}^{-\mathbf{1 / 2}} \mathrm{Q}^{\mathrm{T}}$ is associated with such an affine transformation.

Remark 2. It is obvious that $\gamma_{*}=s_{* 1}^{2}+s_{* 2}^{2}+\ldots+s_{* m}^{2}$, where all the elements of the sum $s_{* i},(i=1,2, \ldots, m)$ are pairwise independent and are the components of vector $\mathbf{S}_{*}=\boldsymbol{\Lambda}^{-\mathbf{1} / \mathbf{2}} \mathrm{Q}^{\mathrm{T}}\left(\Theta_{\mathbf{e}}-\Theta_{*}\right)$ (see above.). As the sample volume is increasing Student distribution asymptotically approaches the standard normal distribution $N(0,1)$. The distribution $\gamma_{*}$, as noted above, asymptotically approaches to $\chi^{2}$ distribution with $m$ degrees of freedom.

Remark 3. The case of indirect parameters estimation through some indirect measurements vector $\mathbf{Y}$ with a linear regression connection $\mathbf{Y}=\mathbf{X} \Theta+\varepsilon$ is also possible.

It tends to assume that the normally distributed vector of measurement errors $\varepsilon$ has zero mathematical expectation and independent components with equal deviations: $\varepsilon \sim \mathbf{N}\left(\mathbf{O}, \sigma^{2} \mathbf{I}\right)$, where $\mathbf{O}$ is a zero vector, $\mathbf{I}$ is an identity matrix. In this case the estimate of the parameter vector $\Theta$ by the least squares method is equal to $\boldsymbol{\Theta}_{\mathbf{e}}=\left(\mathbf{X}^{\mathrm{T}} \mathbf{X}\right)^{-1} \mathbf{X}^{\mathrm{T}} \mathbf{Y}$. The mutual covariance matrix of this estimation has the form $\mathbf{V}_{\boldsymbol{\Theta}}=\sigma_{\mathbf{e}}^{2}\left(\mathbf{X}^{\mathrm{T}} \mathbf{X}\right)^{-1}$, where $\sigma_{e}^{2}=(n-$ $m)^{-1} \sum_{j=1, \ldots, n}\left(y_{j}-\mathbf{X}_{\mathbf{j}} \boldsymbol{\Theta}_{\mathbf{e}}\right)^{2}$ is unbiased estimator $\sigma^{\mathbf{2}}$ (here $\mathbf{X}_{\mathbf{j}}$ is $j$-th row of $\mathbf{X})$. Then the value [12]

$$
\eta_{*}=\gamma_{*} m^{-1}=\sigma_{\mathbf{e}}^{-\mathbf{2}} \mathbf{m}^{-\mathbf{1}}\left(\boldsymbol{\Theta}_{\mathbf{e}}-\boldsymbol{\Theta}_{*}\right)^{\mathrm{T}}\left(\mathbf{X}^{\mathrm{T}} \mathbf{X}\right)\left(\boldsymbol{\Theta}_{\mathbf{e}}-\boldsymbol{\Theta}_{*}\right)
$$

will have exactly $\mathbf{F}$-distribution if the hypothesis $H_{*}: E \boldsymbol{\Theta}_{\mathbf{e}}=\boldsymbol{\Theta}_{*}$ is true. In other words, $\eta_{*} \sim F_{m, n-m}$ and it can be used to test this hypothesis.

Obviously, a divisor of the quadratic form $m$ defining $d_{2}$ does not disturb the metric ratios, or rather, converts the generalized Euclidean metric $d_{2}$ into the equivalent metric $d_{2}^{\prime}=m^{-1 / 2} d_{2}$. Therefore, rejecting the hypothesis $H_{*}$ in accordance with the $\mathbf{F}$-criteria implies rejecting any other hypothesis $H_{\tau}$, that is the hypothesis $H_{c} \omega$. In this case components $s_{i}(i=1,2, \ldots, m)$ of the vector $\mathrm{S}_{*}=\boldsymbol{\Lambda}^{-\mathbf{1} / \mathbf{2}} \mathrm{Q}^{\mathrm{T}}\left(\Theta_{\mathbf{e}}-\Theta_{*}\right)$ have Student distribution with degree of freedom $n-m$.

\section{Illustration of Indirect Parameter Estima- tion}

Consider a linear regression model as a mathematical model of the tested robotic mechanism

$$
y_{i}=\Theta_{0}+\Theta_{1} x_{i 1}+\Theta_{2} x_{i 2}+\epsilon_{1}, \quad i=1,2, \ldots, n .
$$

Here the values of covariates $x_{i 1}$ and $x_{i 2}$ are interpreted as set modes (or load), and the response $y_{i}$ is interpreted as a measured external parameter 
characterizing the overall mechanism state. Parameters $\Theta_{0}, \Theta_{1}$ and $\Theta_{2}$ are interpreted as internal parameters of the mechanism to be an indirect estimation by a series of tests.

Model of indirect parameters estimation for the constructed mechanism is of great interest as it is a more general case of estimation. Recall that averaging direct measurements is the OLS-estimate, as well. Suppose the parameters characterizing response of a tested mechanism to a load are constrained by $0<\Theta_{1}<1$ and $0<\Theta_{2}<1$. Table 1 presents the model data $(\mathrm{n}=33)$ providing the points $\left(\Theta_{1}, \Theta_{2}\right)$ estimated by OLS to be in a specified domain. Note that the transition to the centered values from Table 1 leads to the elimination of uninterested to us (by assumption) parameter $\Theta_{0}$, which is not correlated with the load fluctuations.

Table 1: Input data

\begin{tabular}{|c|c|c|c|c|c|c|c|}
\hline \multirow{2}{*}{$\mathbf{N}$} & Response & \multicolumn{2}{|c|}{ Intensity } & \multirow{2}{*}{$\mathbf{N}$} & Response & \multicolumn{2}{|c|}{ Intensity } \\
\cline { 7 - 8 } & variable $\mathbf{y}$ & $\mathbf{x}_{\mathbf{1}}$ & $\mathbf{\mathbf { x } _ { \mathbf { 2 } }}$ & & variable $\mathbf{y}$ & $\mathbf{x}_{\mathbf{1}}$ & $\mathbf{x}_{\mathbf{2}}$ \\
\hline 1 & 2,870 & 2,570 & 3,090 & 18 & 3,799 & 3,735 & 3,543 \\
\hline 2 & 2,927 & 2,642 & 3,116 & 19 & 3,841 & 3,790 & 3,557 \\
\hline 3 & 3,001 & 2,729 & 3,168 & 20 & 3,879 & 3,840 & 3,573 \\
\hline 4 & 3,062 & 2,795 & 3,213 & 21 & 3,919 & 3,891 & 3,588 \\
\hline 5 & 3,110 & 2,853 & 3,240 & 22 & 3,951 & 3,940 & 3,602 \\
\hline 6 & 3,160 & 2,929 & 3,264 & 23 & 3,987 & 3,986 & 3,615 \\
\hline 7 & 3,220 & 2,995 & 3,293 & 24 & 4,022 & 4,032 & 3,625 \\
\hline 8 & 3,282 & 3,071 & 3,329 & 25 & 4,062 & 4,078 & 3,632 \\
\hline 9 & 3,350 & 3,164 & 3,356 & 26 & 4,095 & 4,124 & 3,638 \\
\hline 10 & 3,421 & 3,266 & 3,380 & 27 & 4,125 & 4,169 & 3,643 \\
\hline 11 & 3,484 & 3,345 & 3,405 & 28 & 4,149 & 4,209 & 3,649 \\
\hline 12 & 3,526 & 3,398 & 3,430 & 29 & 4,172 & 4,248 & 3,654 \\
\hline 13 & 3,577 & 3,453 & 3,449 & 30 & 4,192 & 4,285 & 3,654 \\
\hline 14 & 3,623 & 3,509 & 3,470 & 31 & 4,214 & 4,321 & 3,645 \\
\hline 15 & 3,664 & 3,568 & 3,489 & 32 & 4,231 & 4,357 & 3,637 \\
\hline 16 & 3,716 & 3,626 & 3,507 & 33 & 4,229 & 4,391 & 3,617 \\
\hline 17 & 3,758 & 3,679 & 3,525 & Average & $\mathbf{3 , 6 8 5}$ & $\mathbf{3 , 6 0 6}$ & $\mathbf{3 , 4 7 3}$ \\
\hline
\end{tabular}

The OLS-estimates of the parameters are calculated in accordance with Table 1. Solving Lagrange Problems for each of the four constraints gives only two points of maximum likelihood $(=3,41 E-05)$ at the lower boundary and $(=3,99 E-06)$ on the upper boundary ( Table 2 and Figure 1$)$. The solution at the boundary $\Theta_{1}=0$ gives the value $\Theta_{2}=2,42$, and at the boundary $\Theta_{1}=1$ it is $\Theta_{2}=-0,72$. These values do not satisfy conditions $0<\Theta_{2}<1$ 
and are not considered further. Point at the lower boundary gives the greatest value of the likelihood $(3,41 E-05)$.

On the following figure:

1. OLS-estimate;

2. point of maximum likelihood at the lower boundary;

3. point of maximum likelihood at the upper boundary;

4. nearest boundary point.

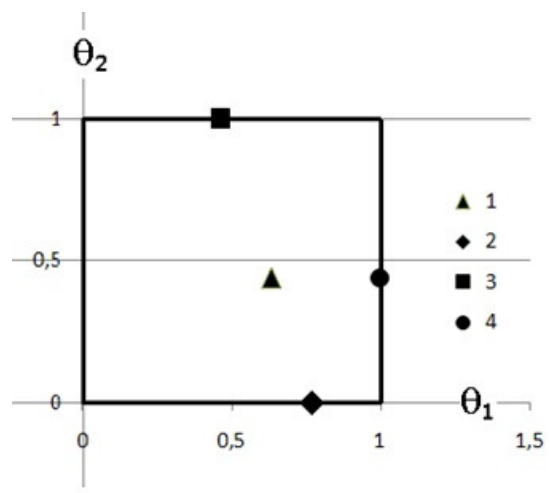

Figure 1: The geometrical arrangement of the parameter estimates

Table 2: Statistical reliability test

\begin{tabular}{|c|c|c|c|c|c|c|}
\hline Type of Parameter & \multicolumn{2}{|c|}{ Parameters } & \multicolumn{4}{|c|}{ Statistics } \\
\cline { 5 - 8 } Estimation & $\boldsymbol{\Theta}_{\mathbf{1}}$ & $\boldsymbol{\Theta}_{\mathbf{2}}$ & $\begin{array}{c}\boldsymbol{\chi}^{\mathbf{2}} \\
(\boldsymbol{\gamma})\end{array}$ & $\begin{array}{c}\mathrm{F} \\
(\boldsymbol{\eta})\end{array}$ & $\left|s_{1}\right|$ & $\left|s_{2}\right|$ \\
\hline OLS-Estimate & 0,63 & 0,44 & - & - & - & - \\
\hline $\begin{array}{c}\text { MLP at the } \\
\text { Lower Boundary }\end{array}$ & 0,77 & 0,00 & 6,93 & 3,46 & 2,63 & 0,05 \\
\hline $\begin{array}{c}\text { MLP at the } \\
\text { Upper Boundary }\end{array}$ & 0,46 & 1,00 & 11,22 & 5,61 & 3,35 & 0,06 \\
\hline $\begin{array}{c}\text { Nearest Boundary } \\
\text { Point }\end{array}$ & 1,00 & 0,44 & 1294,54 & 647,27 & 0,62 & 35,97 \\
\hline Critical Values $(\alpha=0,05):$ & $\chi_{2}^{2}(\alpha)=5,99 ; F_{2,30}(\alpha)=3,32 ; t_{30}^{\alpha / 2}=2,36$ \\
\hline
\end{tabular}

Table 2 illustrates the results of the statistical test. For comparison the table includes the point with maximum likelihood at the upper boundary and 
geometrically closest boundary point, which is at the right boundary (Figure $1)$.

Here MLP is maximum likelihood point.

As shown in Table 2, the point of maximum likelihood provides test values that is in excess of the critical parameters values (as the data was modeled initially). The other two boundary points provide test values that are more in excess of critical ones. This fact illustrates the given example. For points being beyond the domain of parameters acceptable region, the result is analogical.

\section{Conclusion}

In the paper we propose the procedure estimating the parameters of the experimental robots sample in a series of tests. The procedure allows rejecting the samples with the parameters unsuitable for the constructed sample. The algorithm may be used effectively in the processes of designing manipulator robots $[4,11,12,13]$.

It is illustrated that when the constructed robotic mechanism with a large number of parameters is tested, it may be necessary to test a complex hypothesis, which arises from the restrictions imposed on the parameters. It is also shown that the test process of this complex hypothesis is reduced to testing a simple hypothesis about the boundary point providing maximum likelihood. This fact and the proposed algorithm significantly expands opportunities of the adequate testing of complex robotic mechanisms and multivariable systems, increasing their reliability.

\section{References}

[1] G. V. Alferov, O. A. Malafeyev, A. S. Maltseva, Game-theoretic model of inspection by anti-corruption group, International Conference on $\mathrm{Nu}$ merical Analysis and Applied Mathematics 2014, ICNAAM 2014, Greece, 1648 (2015). http://dx.doi.org/10.1063/1.4912668

[2] G. V. Alferov, O. A. Malafeyev, A. S. Maltseva, Programming the robot in tasks of inspection and interception, 2015 International Conference on Mechanics - Seventh Polyakhov's Reading, Saint Petersburg State University, St. Petersburg, (2015).

http://dx.doi.org/10.1109/polyakhov.2015.7106713

[3] P. Efimova, D. Shymanchuk, Dynamic model of space robot manipulator, Applied Mathematical Sciences, 9 (2015), no. 94, 4653-4659.

http://dx.doi.org/10.12988/ams.2015.56429 
[4] A. V. Kondrashkov, Yu. A. Pichugin, On the Identification and statistical testing stability of Volterra model, St.-Petersburg Polytechnical University J., Physics and Mathematics, 1 (2014), no. 189, 124-135.

[5] F. Kulakov, G. Alferov, P. Efimova,Methods of remote control over space robots, 2015 International Conference on Mechanics - Seventh Polyakhov's Reading, Saint Petersburg State University, St. Petersburg, (2015). http://dx.doi.org/10.1109/polyakhov.2015.7106742

[6] F. Kulakov, G. Alferov, P. Efimova, S. Chernakova, D. Shymanchuk, Modeling and control of robot manipulators with the constraints at the moving objects, 2015 International Conference "Stability and Control Processes" in Memory of V.I. Zubov (SCP), St. Petersburg, (2015), 102-105. http://dx.doi.org/10.1109/scp.2015.7342075

[7] O. Malafeyev, G. Alferov, M. Andreyeva, Group strategy of robots in game-theoretic model of interception with incomplete information, 2015 International Conference on Mechanics - Seventh Polyakhov's Reading, Saint Petersburg State University, St. Petersburg, (2015), 1-3.

http://dx.doi.org/10.1109/polyakhov.2015.7106751

[8] O. A. Malafeyev, S. A. Nemnyugin, G. V. Alferov, Charged particles beam focusing with uncontrollable changing parameters, 2014 2nd International Conference on Emission Electronics ICEE, St. Petersburg, (2014), 1-3. http://dx.doi.org/10.1109/emission.2014.6893964

[9] O. A. Malafeyev, E. G. Neverova, S. A. Nemnyugin, G. V. Alferov, Multicriteria model of laser radiation control, 2014 2nd International Conference on Emission Electronics, ICEE 2014, St. Petersburg, (2014), 1-5. http://dx.doi.org/10.1109/emission.2014.6893966

[10] O. A. Malafeyev, N. D. Redinskikh, G. V. Alferov, Electric circuits analogies in economics modeling: Corruption networks, 2014 2nd International Conference on Emission Electronics, ICEE 2014, St. Petersburg, (2014), 1-5. http://dx.doi.org/10.1109/emission.2014.6893965

[11] Yu. A. Pichugin, Geometrical aspects of testing the complex statistical hypothesis in mathematical simulation, St. Petersburg Polytechnic University Journal (and Hosting by Elsevier B. V.), Physics and Mathematics, 218 (2015), no. 2, 123-137. http://dx.doi.org/10.5862/jpm.218.13

[12] Yu. A. Pichugin, The testing of complex statistical hypothesis in linear regression analysis, Handbook "Nauchnaya sessiya GUAP", St. Petersburg: GUAP, 1 (2014), 275-276. 
[13] Yu. A. Pichugin, A. V. Kondrashkov, The probabilistic estimate of the stability and hazard of the dynamics of a space object on the basis of Keplers model, Handbook "Nauchnaya sessiya GUAP", St. Petersburg: GUAP, 1 (2014), 273-274.

[14] A. Shmyrov, V. Shmyrov, D. Shymanchuk, Prospects for the use of space robots in the neighbourhood of the libration points, Applied Mathematical Sciences, 8 (2014), no. 50, 2465-2471.

http://dx.doi.org/10.12988/ams.2014.43158

[15] A. Shmyrov, D. Shymanchuk, Maneuvering in near-Earth space with the use of the collinear libration points, 2015 International Conference on Mechanics - Seventh Polyakhov's Reading, Saint Petersburg State University, St. Petersburg, (2015), 1-5.

http://dx.doi.org/10.1109/polyakhov.2015.7106777

[16] A. Shmyrov, D. Shymanchuk, L. Sokolov, The interception problem of a celestial body with the use of the collinear libration points, 2015 International Conference "Stability and Control Processes" in Memory of V.I. Zubov (SCP), St. Petersburg, (2015), 129-131. http://dx.doi.org/10.1109/scp.2015.7342068

Received: December 26, 2015; Published: February 11, 2016 